\section{Nicotine, tobacco and addiction}

SIR - For many people, the concept of addiction involves taking of drugs'; most official definitions of addiction include drug ingestion. Despite such definitions, there are many potentially addictive behaviours that do not involve drug ingestion, for example gambling, overeating, sex, exercise, computer-game playing, the Internet, pair bonding and work ${ }^{2,3}$. Such diversity has led to all-encompassing definitions of what constitutes addictive behaviour. Trying to define 'addiction' is rather like defining a 'mountain' or 'tree' in that there is no single set of criteria that can ever be necessary or sufficient to define all instances. In essence, the whole is easier to recognize than the parts. Here I suggest six components that in my view need to be fulfilled if a behaviour is to be defined as 'addictive'.

Salience: when the particular activity becomes the most important activity in people's lives and dominates their thinking (preoccupations and cognitive distortions), feelings (cravings) and behaviour (deterioration of socialized behaviour). For instance, even if they are not actually engaged in the behaviour, they will be thinking about the next time they will be. Mood modification: subjective experiences that people report as a consequence of engaging in the particular activity and can be seen as a coping strategy (they experience an arousing 'buzz' or a 'high' or a paradoxically tranquillizing feel of 'escape' or 'numbing').

Tolerance: a process whereby increasing amounts of the particular activity are required to achieve the former effects. For instance, a gambler may have gradually to increase the size of the bet to experience a euphoric effect that was initially obtained by a much smaller bet.

Withdrawal symptoms: unpleasant feeling states and/or physical effects that occur when the particular activity is discontinued or suddenly reduced, for example 'the shakes', moodiness or irritability.

Conflict: conflicts between addicts and those around them (interpersonal conflict) or from within the individual (intrapsychic conflict) that are concerned with the particular activity.

Relapse: the tendency for repeated reversions to earlier patterns of the particular activity to recur and for even the most extreme patterns typical of the height of the addiction to be quickly restored after many years of abstinence or control.

I believe that explanations for addiction must come from a biopsychosocial approach, in that 'addiction' arises from a combination of biological predisposition, social environment and psychological constitution. To many, this goes without saying, but others present oversimplistic and parsimonious explanations. Behaviorial addictions do exist, and should be treated no differently from the better-known chemically based addictions.

\section{Mark Griffiths}

Psychology Division,

Nottingham Trent University,

Nottingham NG1 4BU, UK

1. Rachlin, H. Psychot. Sci. 1, 294-297 (1990)

2. Miller, W. R. The Addictive Behaviors (Pergamon, Oxford, 1980)

3. Orford, J. Excessive Appetites: A Psychological View of the Addictions (Wiley, Chichester, 1985).

SIR - Although there is little doubt that cigarette smoking is hazardous to health and that nicotine plays a major role in reinforcing this behaviour ${ }^{1}$, we believe that certain effects of nicotine itself need to be dissociated from tobacco and other drugs of abuse.

Despite recent findings ${ }^{2}$ demonstrating common neuropharmacological and neuroanatomical similarities between the effects of nicotine and other drugs of abuse, there are some important differences. For example, nicotine is typically devoid of the profound euphoric and perceptual effects offered by many drugs of abuse, and there is little evidence that the habitual consumption of nicotine (via tobacco) causes depression or psychosis ${ }^{3}$.

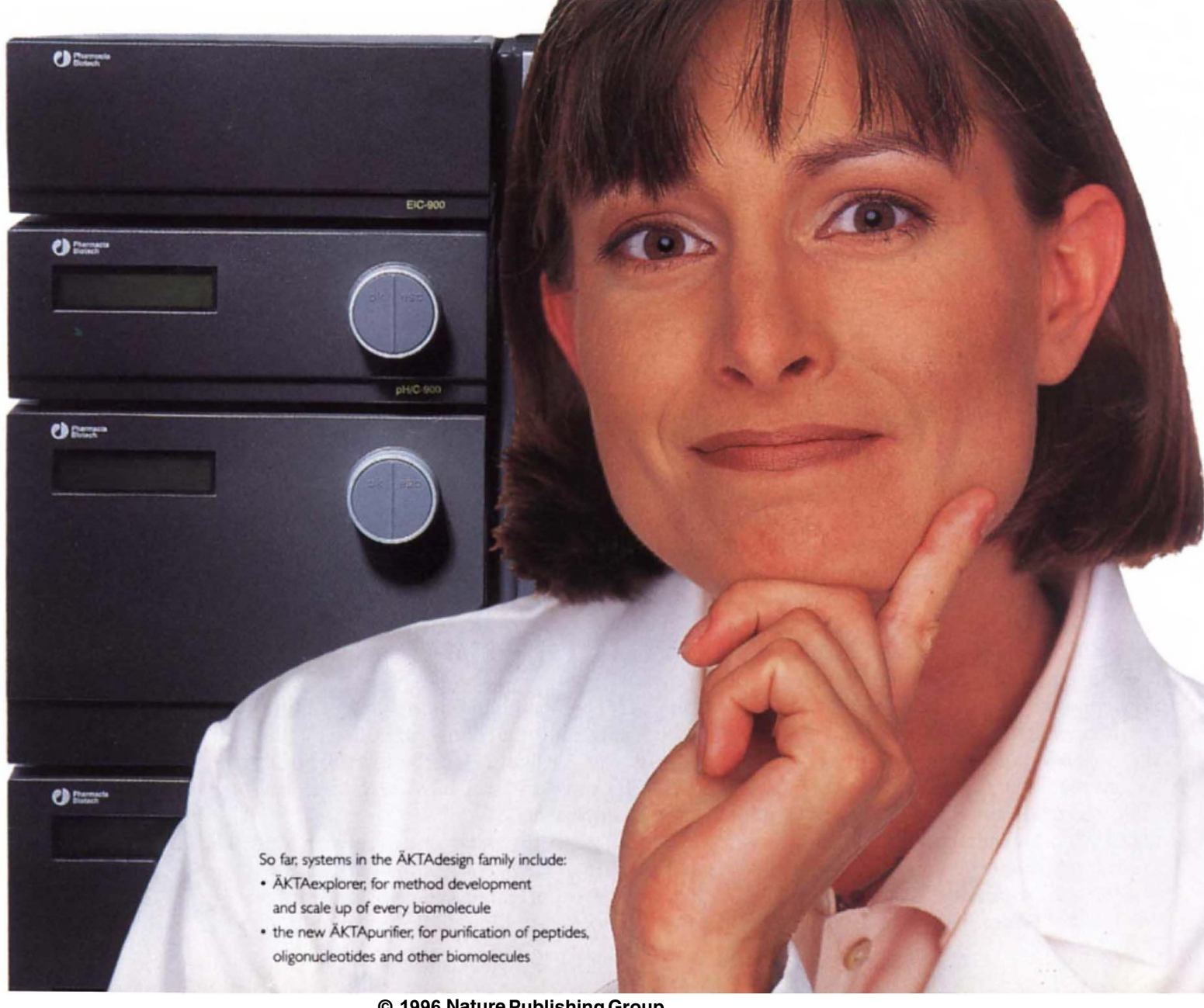


Unfortunately, the title of the Nature News and Views article ${ }^{4}$ about the new findings read "Smoking... harmful to the brain", when there was little if any evidence presented to suggest that nicotine itself is harmful to the brain. Although tobacco contains nicotine, it also contains many harmful chemicals in the tar and smoke. It is likely that the increase in cancers and other diseases among smokers is due in large part to the many non-nicotine substances found in cigarette smoke ${ }^{1}$.

The smoker's habit is very complex and is associated with many factors. These include flavour, smoke, personality, mood and the social setting one happens to be in, which may involve having an alcoholic drink, being under stress or, among other things, having fun. Perhaps most important, however, is the rapid rise in blood levels of nicotine associated with inhalation of tobacco smoke $^{2}$. Indeed, an important

1. The Health Consequences of Smoking: Nicotine Addiction, Report of the Surgeon General, Office on Smoking and Health (Government Printing Office, Washington DC, 1988).

2. Pontieri, F. E., Tanda, G.. Orzi, F. \& Di Chiara, G. Nature 382, 255 (1996).

3. Warturton, D. M. in Effects of Nicotine on Biological Systems II (eds Clarke, P. B. S. et al.) 257-264 (Birkhauser, Basel and Boston, 1995).

4. Iversen, L. Nature 382, 206 (1996).

5. Thomas, G. A. O. et al. N. Engl. J. Med. 332, 988-992 (1995).

6. Sanberg. P. R. et al. Pharmacol. Ther. (in the press).

7. Levin, E. D. et al. Psychopharmacology 123, 55-63 (1996). characteristic of all drugs that produce dependency is the time between behavioural administration (smoking a cigarette) and the drug's entry into the brain.

The US Food and Drug Administration has recently approved the over-the-counter sale of transdermal nicotine as a smoking cessation aid, a drug delivery system designed to avoid the harmful chemicals found in cigarette smoke. The slower absorption of nicotine offered by the transdermal nicotine patch relative to tobacco products should substantially reduce the likelihood of nicotine dependence in users of the patch. For example, a recent doubleblind, placebo-controlled study investigating the therapeutic potential of the transdermal nicotine patch for ulcerative colitis found little evidence for nicotine withdrawal symptoms following discontinuation of the patch, despite 26 weeks of daily applications of $15-\mathrm{mg}$ nicotine patches ${ }^{5}$.

Cigarette smoking is clearly a major health problem, and decreasing its use is a worldwide effort, but there is also a growing body of evidence to indicate that nicotine has therapeutic properties. Nicotine improves cognitive function and may reduce the symptoms of certain neuropsychiatric disorders such as Tourette's syndrome and attention deficit/hyperactivity disorder ${ }^{6,7}$. But many of these efforts will be hampered if the role of nicotine in cigarette smoking is overemphasized without acknowledging the essential differences between nicotine, tobacco and other drugs of abuse.

\section{R. Doug Shytle}

Archie A. Silver

Department of Psychiatry,

Paul R. Sanberg*

Department of Surgery, MDC-16,

University of South Florida

College of Medicine,

12901 Bruce B. Downs Blvd,

Tampa, Florida 33612-4799, USA

email: psanberg@com1.med.usf.edu

*To whom correspondence should be addressed.

\section{Strange characters}

Sir - J. T. C. Sellick (Nature 383, 569; 1996) raises the issue of what a researcher has to do when he/she changes his/her name. The answer is simply to start using your newly acquired name.

There are some more important name issues, for example authors with names contain non-ASCII letters. We often see that our names change to accommodate the country we happen to live in. Many variations can therefore be found on various databases; a standard is not going to happen until we all speak the same language.

\section{Keld Sorensen}

Pierce Chemical Co., PO Box 117,

Rockford, Illinois 61105, USA

e-mail:Kelds@uic.edu

\title{
ÄKTAdesign:
}

\section{an open purification platform for $\mathbf{a l 1}$ of your biomolecules}

What type of purification is going on in your lab? Do some of your colleagues develop methods and optimize schemes to purify peptides, proteins, or oligonucleotides at every purification scale? Are others purifying natural, synthetic and recombinant peptides? Are yet others purifying native or recombinant proteins? Or perhaps you do all of this yourself.

Doing individual types of purification has meant following individual working procedures - until now, that is. Until ÄKTA ${ }^{T M}$ design ( $̈$ KTA is the Swedish word for real; it's pronounced eckta).

\section{With ÄKTAdesign, your purification Systems won't act like strangers to one another}

\begin{abstract}
ÄKTAdesign is the name of a new platform for a family of purification systems and pre-packed columns exclusively from us, Pharmacia Biotech. The platform integrates fully-biocompatible hardware solutions with a control system that gives you control over purification systems from lab to production scales. It lets everyone use the same better, smarter way of doing purification. All of which means you can operate every ÄKTAdesign system once you've used any one of them.

Each ÄKTAdesign system lets you use pre-set protocols that automatically resolve all major purification tasks-including automatic method scouting. Each system gives you pre-set running parameters for most purification techniques. Each system is supported with an extensive range of technique-specific, pre-packed columns. Each system automatically prepares buffers from stock solutions — without manual titration. And each system operates via UNICORN — with this single control system, you can instantly transfer your methods to purification systems at all scales.

What does your lab want to purify today? A version of ÄKTAdesign will suit all your needs. Call us: I (800) 5263593 from the USA; +8I (0)3 34926949 from Japan; or +46 (0) I8 I6 50 II from Europe and the rest of the world. Ask for a free brochure. Or meet us on the Internet at http://wnww.biotech.pharmacia.se.
\end{abstract}

